\title{
Sensor Fusion with Extended Kalman Filter Applied to Indoor Tracking: Comparing RSSI and RTT
}

\author{
R. C. Nascimento* T. P. Chagas* \\ * Laboratório de Mecatrônica, Universidade Estadual de Santa Cruz, \\ Rodovia Jorge Amado, Km 16, Ilhéus - BA, CEP 45662-900 \\ (e-mails: rafacardoso8@hotmail.com,tpchagas@uesc.br)
}

\begin{abstract}
The following work aims at presenting the proposal of using Extended Kalman Filter (EKF) sensor fusion applied to the indoor tracking and navigation issue. Two sensor fusion approaches were proposed. The first one called IMU+RSSI fuses the acceleration data of an Inertial Measurement Unit (IMU) with Received Signal Strength Indicator (RSSI) data provided by wireless access points. The second one called IMU+RTT fuses IMU data and distance measurements provided by access points using Round Trip Time (RTT). The resultant trajectories given by both fusion methods are compared to a reference path through simulations results based on real sensor characteristics. As expected, the IMU+RTT fusion showed better results, even considering multirate sensors. The proposed methods are promising to implement tracking and navigation systems in indoor environments.
\end{abstract}

Keywords: Extended Kalman Filter; RSSI; RTT; sensor fusion

\section{INTRODUCTION}

The Global Positioning System (GPS) is the current technology used for outdoor navigation. However, for indoor environments this technology is not useful, once the GPS signal is unable to reach inside buildings and others indoor environments in general (Zhang et al., 2010). There are several applications for indoor tracking and navigation systems such as tracking of autonomous vehicles and mobile robots, asset tracking in warehouses or human navigation in large indoor environments (e.g malls, airports, hospitals) (Kumkeaw et al., 2013).

Even with the large amount of research in this area in the past few decades, the indoor tracking is still an open issue. The current methods are mostly imprecise and with almost none practical use. These methods usually consist in read sensors data and apply mathematical methods for convert this information (e.g acceleration, signal strength, round trip time) in position (Jin et al., 2018).

There are two common approaches in terms of sensors: the relative position or inertial sensors and the absolute position sensors. Inertial navigation usually is supported by an Inertial Measurement Unit (IMU) (Jin et al., 2018). An IMU generally consists in an embedded system containing an accelerometer and a gyroscope. The linear acceleration measurements are integrated twice yielding in position and the self-contained feature allows tracking without an external reference. However, the integration of measurements subjected to random noises leads to a drift in the trajectory over time, called random walk. This leads to unacceptable errors for almost every tracking application (Borenstein et al., 1997).
On the other hand, the absolute position sensors provide information of distance to a known position reference (Kumkeaw et al., 2013). For information about absolute position it is required a minimum of three measurements related to reference points. These methods are also known as trilateration (Santana et al., 2017). The usual approach for trilateration methods is Received Signal Strength Indicator (RSSI) based. The main idea of RSSI trilateration is to measure the signal strength from multiple wireless access points (APs) and use a wave propagation model to relate the RSSI measurements with the distance between the current position and the APs. With these distances it is possible to track the current target position (Borenstein et al., 1997).

The wave propagation model is an over-simplification which does not take account multipath fading of the signal (Mao et al., 2007). Then, most of times the RSSI based techniques are inaccurate and with no practical use when considered alone. For more precise information about absolute position, time-based methods were developed over the years. The Time-of-Arrival (TOA) is a common proposed time-based method. It consists in measuring the time propagation of the waves and multiply it the speed of light $c$. However, this approach requires highly resolute time information and precise time synchronization (Park et al., 2007).

The Round Trip Time (RTT) is a time-based method inspired in a variation of TOA, the Time-Difference-ofArrival (TDOA). The RTT does not require time synchronization and with an appropriate hardware it is possible to measure the distance between a node (e.g smartphones, wireless stations) and the APs. The RTT approach is much more precise than the RSSI based methods, Google affirms 
that 1-2 meter accuracy is possible, instead of typical 3-4 meters of the RSSI based tracking (Want et al., 2018).

A hardware with RTT support was not feasible until 2018, when Google released the Android Pie 9.0 with RTT support, which opened a new perspective in indoor traking and navigation problem (Want et al., 2018). The RTT technology is supported by IEEE $802.11-\mathrm{REV} \mathrm{mc}^{2}$ protocol of December 2016. Nowadays wireless technology is in everywhere available for RSSI based traking, and infrastructure implementation is not needed for establish indoor navigation systems in places like airports, malls and another large areas. Also, the embedded system which carry the algorithm does not need to connect to the Wireless networks of the environment, increasing the users privacy and security. In the future, is expected the replacement of APs for RTT supported, allowing indoor tracking for small areas.

The current work aims at reaching even better results using RTT technology through sensor fusion, it is expected to obtain smaller error conditions and allow indoor tracking in small environments like building rooms. The Extended Kalman Filter (EKF) is a common method for nonlinear sensor fusion and over the years was used for fuse inertial position measurements with absolute position information (Jin et al., 2018). This work proposes to simulate an IMU+RTT fusion and compare with an IMU+RSSI fusion. This aims at showing the efficiency of the nonlinear fusion for indoor tracking and the future perspectives of RTT popularization. Yet, simulations considering sensors with different sampling rates are proposed. This multirate sensors insert a real application problem in the results.

The paper is organized as follows. Section 2 gives an overview about the EKF and its equations. Section 3 shows the considered trajectory and the system model, as the fusion strategies. Section 4 bring results and discussions and Section 5 gives the conclusion of the work.

\section{EXTENDED KALMAN FILTER (EKF)}

Kalman Filter (KF) is an optimal estimator based on leastsquares (Brown, 2012). The KF is capable of estimate the system state and output based on system model and measurement $z_{k}$ subjected to gaussian noises. For nonlinear systems the Extended Kalman Filter is used, provided that the functions are differentiable and almost linear for short intervals (Julier and Uhlmann, 1997).

The EKF inputs, $u_{k}$ and $z_{k}$, are respectively system inputs (e.g acceleration, wheel command of a robot, angle, etc) and measurements, which are function of the state variables. The EKF output are the estimated state, $\hat{x}_{k}$, and output $\hat{z}_{k}$. For a possibly nonlinear system the process model and measurement model are given respectively by

$$
\begin{gathered}
\mathbf{x}_{k}=f\left(\mathbf{x}_{k-1}, \mathbf{u}_{k}\right)+\mathbf{w}_{k} \\
\mathbf{z}_{k}=h\left(\mathbf{x}_{k}, \mathbf{u}_{k}\right)+\mathbf{v}_{k}
\end{gathered}
$$

with $x: N \rightarrow R^{n}, u: N \rightarrow R^{m}, f: R^{n} \times R^{m} \rightarrow R^{n}, z:$ $N \rightarrow R^{l}, f: R^{n} \times R^{m} \rightarrow R^{l}, n, m, l \in N, w_{k} \sim \mathcal{N}\left(0, \mathbf{Q}_{k}\right)$ and $v_{k} \sim \mathcal{N}\left(0, \mathbf{R}_{k}\right)$.
Given $\hat{x}_{0}$ and $P_{0}$, the filter application consists of two steps: the predict phase (1a) and (2a), and the update phase (2b-2f) (Ristic et al., 2003).

$$
\begin{gathered}
\mathbf{P}_{k \mid k-1}=\mathbf{F}_{k} \mathbf{P}_{k-1 \mid k-1} \mathbf{F}_{k}^{T}+\mathbf{Q}_{k} \\
\tilde{\mathbf{y}}_{k}=\mathbf{z}_{k}-h\left(\hat{\mathbf{x}}_{k \mid k-1}, \mathbf{u}_{k}\right) \\
\mathbf{S}_{k}=\mathbf{H}_{k} \mathbf{P}_{k \mid k-1} \mathbf{H}_{k}^{T}+\mathbf{R}_{k} \\
\mathbf{K}_{k}=\mathbf{P}_{k \mid k-1} \mathbf{H}_{k}^{T} \mathbf{S}_{k}^{-1} \\
\hat{\mathbf{x}}_{k \mid k}=\hat{\mathbf{x}}_{k \mid k-1}+\mathbf{K}_{k} \tilde{\mathbf{y}}_{k} \\
\mathbf{P}_{k \mid k}=\left(\mathbf{I}-\mathbf{K}_{k} \mathbf{H}_{k}\right) \mathbf{P}_{k \mid k-1}
\end{gathered}
$$

The external measurement is $z_{k}$ and $P: N \rightarrow R^{n \times n}$ is the covariance error. Equations (3a) and (3b) indicate how to calculate the Jacobian matrices of the functions $f$ and $h$. (Julier and Uhlmann, 1997).

$$
\begin{aligned}
& \mathbf{F}_{k}=\left.\frac{\partial f(k, \mathbf{x}))}{\partial \mathbf{x}}\right|_{\mathbf{x}=\hat{\mathbf{x}}_{k}} \\
& \mathbf{H}_{k}=\left.\frac{\partial h(k, \mathbf{x}))}{\partial \mathbf{x}}\right|_{\mathbf{x}=\hat{\mathbf{x}}_{k}}
\end{aligned}
$$

\section{METHODOLOGY}

This section presents the work methodology based on simulations executed in MATLAB. We emphasis that nowadays acquiring equipment to apply RTT is not viable in Brazil.

\subsection{Trajectory and Kinematic model}

Figure 1 shows the square trajectory of $5 \mathrm{~m}$ x $5 \mathrm{~m}$ drawn on MATLAB used as case study. Also, it was generated a time vector from $t=0$ to $t=30$ s in which this trajectory must be fulfilled. The process of derive numerically two times the points of this trajectory with respect to time results in the acceleration vector of the movement $\mathbf{u}_{k}=\left[\begin{array}{ll}a_{x k} & a_{y k}\end{array}\right]$ (Nussenzveig, 2013).

The system governing this movement is linear and can be written in the state space form according to (4) (Kumkeaw et al., 2013).

$$
\begin{aligned}
{\left[\begin{array}{c}
x_{k} \\
y_{k} \\
\dot{x}_{k} \\
\dot{y}_{k}
\end{array}\right]=} & {\left[\begin{array}{cccc}
1 & 0 & T_{i m u} & 0 \\
0 & 1 & 0 & T_{i m u} \\
0 & 0 & 1 & 0 \\
0 & 0 & 0 & 1
\end{array}\right]\left[\begin{array}{c}
x_{k-1} \\
y_{k-1} \\
\dot{x}_{k-1} \\
\dot{y}_{k-1}
\end{array}\right]+} \\
& {\left[\begin{array}{cc}
\frac{1}{2} T_{i m u}^{2} & 0 \\
0 & \frac{1}{2} T_{i m u}^{2} \\
T_{i m u} & 0 \\
0 & T_{i m u}
\end{array}\right]\left[\begin{array}{c}
a_{x_{k}} \\
a_{y_{k}}
\end{array}\right]+\mathbf{w}_{k} }
\end{aligned}
$$

Where $\mathbf{x}_{k}=\left[\begin{array}{llll}x_{k} & y_{k} & \dot{x}_{k} & \dot{y}_{k}\end{array}\right]^{T}$ is the state vector and $\left(x_{k}, y_{k}\right)$ indicates the current position on the trajectory and $\left(\dot{x}_{k}, \dot{y}_{k}\right)$ derivates in time. The input is $\mathbf{u}_{k}=\left[\begin{array}{ll}a_{x_{k}} & a_{y_{k}}\end{array}\right]^{T}$, $a_{x}$ and $a_{y}$ accelerations on $x$ and $y$ directions, respectively. 


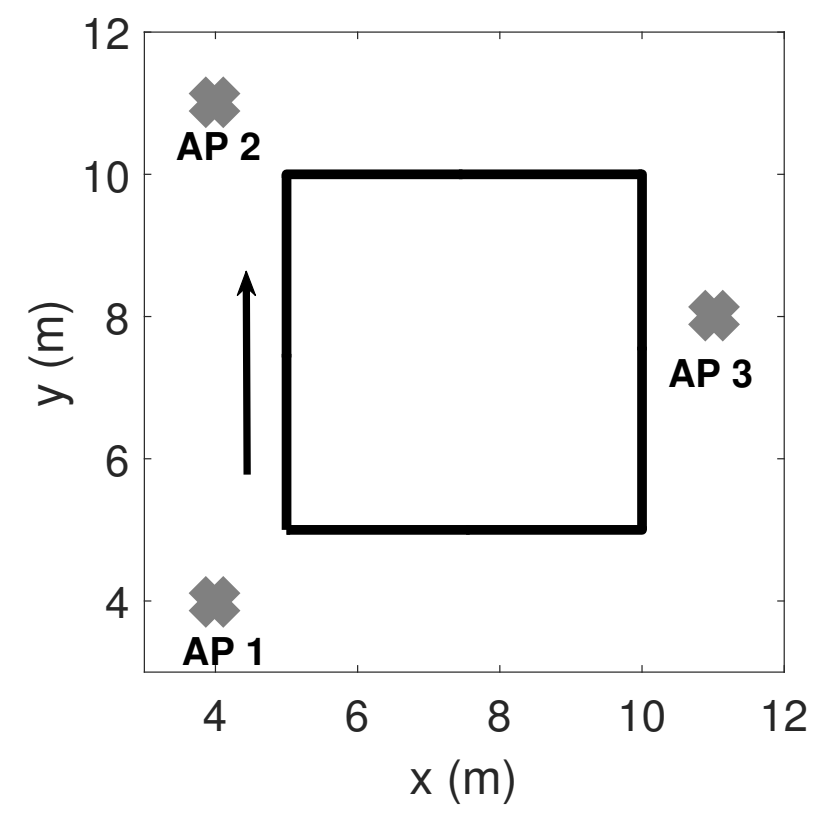

Figure 1. Reference path

The sampling period is $T_{i m u}=0.1$, the IMU sampling period.

Given the position of the APs in the coordinates $(4,4)$, $(4,11)$ and $(11,8)$, it was possible to simulate the RTT measurements, in meters, and RSSI measurements, in $d B m$, for each sampling period.

\subsection{Case studies}

Aiming at compare the efficiency of IMU+RTT against IMU+RSSI fusion, three cases were considered. In cases 1 and 2 , absolute sensor sample rate $T_{a b s}=T_{i m u}$.

- Case 1: In the first case gaussian noises with zero mean and same standard deviations were incorporated in the RTT and RSSI measurements, aiming at showing which fusion method brings better results in similar error conditions.

- Case 2: The second case considers typical standard deviations found in literature for each distance measurement method (Ryo Saito, 2017; Want et al., 2018).

- Case 3: The third case will consider the same standard deviations of case 2 with different sampling periods for each sensor to incorporate the multirate sensors problem. The relation between the sampling period of the absolute position sensors (RTT and RSSI) and IMU is $T_{a b s}=10 T_{i m u}$.

Although the statistical moments of the real world measurements possibly be 2-order above, we consider in this work that the variances $\sigma$ does not vary in time. The standard deviations used in cases 1 to 3 are shown in Table 1 .

\begin{tabular}{c|c|c|c} 
& $R T T$ & $R S S I$ & $I M U$ \\
\hline$\sigma_{1}$ & 1 & 1 & 0.05 \\
\hline$\sigma_{2}$ & 1.5 & 3 & 0.05 \\
\hline$\sigma_{3}$ & 1.5 & 3 & 0.05
\end{tabular}

Table 1. Standard deviations in meters for cases 1 to 3

\subsection{EKF Fusion}

This section shows the fusion strategy for both IMU+RSSI and IMU+RTT cases.

$\boldsymbol{I M U}+\boldsymbol{R T T}: \quad$ The EKF is responsible to merge sensors data. The system model (1a) is represented by (4). While the measurement model (1b) its represented by (5).

$$
h\left(\mathbf{x}_{k}, \mathbf{u}_{k}\right)=h\left(\mathbf{x}_{k}\right)=\overbrace{\left[\begin{array}{l}
\sqrt{\left(x_{1}-x_{k}\right)^{2}+\left(y_{1}-y_{k}\right)^{2}} \\
\sqrt{\left(x_{2}-x_{k}\right)^{2}+\left(y_{2}-y_{k}\right)^{2}} \\
\sqrt{\left(x_{3}-x_{k}\right)^{2}+\left(y_{3}-y_{k}\right)^{2}}
\end{array}\right]}^{\text {Euclidean distance }}+\mathbf{v}_{k}
$$

Where $\left(x_{1}, y_{1}\right),\left(x_{2}, y_{2}\right)$ and $\left(x_{3}, y_{3}\right)$ are coordinates of the APs. Each equation is the euclidean distance between the APs and the current position computed by acceleration measurements using (4). The EKF compares this output with the RTT measurements $z_{k}(2 \mathrm{~b})$ and ponder this values (2e) for a more precise result.

$\boldsymbol{I M U}+\boldsymbol{R} \boldsymbol{S S I :}$ The IMU+RSSI fusion works similar to the previous one. It diverges only in the output function (6) which transforms the state variables $x_{k}$ and $y_{k}$ obtained by (4) in a correspondent RSSI value for each AP. This value will be compared to the measured RSSI values $z_{k}$ on the EKF update phase (2b).

$$
\begin{array}{r}
h\left(\mathbf{x}_{k}, \mathbf{u}_{k}\right)=h\left(\mathbf{x}_{k}\right)= \\
{\left[\begin{array}{l}
P_{\text {ref }}-10 \alpha \log _{10} \sqrt{\left(x_{1}-x_{k}\right)^{2}+\left(y_{1}-y_{k}\right)^{2}} \\
P_{\text {ref }}-10 \alpha \log _{10} \sqrt{\left(x_{2}-x_{k}\right)^{2}+\left(y_{2}-y_{k}\right)^{2}} \\
P_{\text {ref }}-10 \alpha \log _{10} \sqrt{\left(x_{3}-x_{k}\right)^{2}+\left(y_{3}-y_{k}\right)^{2}}
\end{array}\right]+\mathbf{v}_{k}}
\end{array}
$$

The equations show the path-loss model for each AP, which model the behavior of the signal strength of a radius antenna propagation. The constant $P_{\text {ref }}=-53.2161 \mathrm{dBm}$ is the signal strength measured one meter away from the AP, this value was chosen from a previous practical experiment using a real wireless access point. The pathloss exponent $\alpha$ is a constant which express the loss of signal (Mao et al., 2007). One typical value for indoor environments its $\alpha=3.5$ (Miranda et al., 2013). For simplicity, in both cases is considered $\mathbf{v}_{k}=0$, which is reasonable when the APs positions are well know. Orientation is not considered in the models, for application in robotics is easily possible to estimate it by the IMU. Observe that due to (5) and (6) nonlinearities, the classical KF can not be applied and EKF is the chosen alternative. For both fusions, the output is the estimated state vector $\hat{\mathbf{x}}_{k}=\left[\begin{array}{llll}\hat{x}_{k} & \hat{y}_{k} & \hat{\dot{x}}_{k} & \hat{\dot{y}}_{k}\end{array}\right]^{T}$. 
Covariances: Covariance matrices $\mathbf{Q}_{k}$ and $\mathbf{R}_{k}$ of the random noise sequences $w_{k}$ and $v_{k}$ have a important role in the EKF. Basically, this matrices are weights which ponder the trust in the model and the measurements, respectively. The relation between $\mathbf{Q}_{k}$ and $\mathbf{R}_{k}$ in the trust of model and measurement are seen below.

$$
\begin{array}{r}
\uparrow Q_{k} \Rightarrow \text { Trust in the measure } \\
\downarrow R_{k} \Rightarrow \text { Trust in the measure } \\
\downarrow Q_{k} \Rightarrow \text { Trust in the model } \\
\uparrow R_{k} \Rightarrow \text { Trust in the model }
\end{array}
$$

For cases 1 and 2, this matrices were found empirically with values shown in $(7)$.

$$
\begin{array}{r}
\mathbf{Q}_{k}=\mathbf{I}(4) \\
\mathbf{R}_{k}=10^{5} \mathbf{I}(3)
\end{array}
$$

Where $\mathbf{I}$ is the identity matrix.

When we consider multirate sensors, varying the covariance matrices is needed according to the availability of sensors data (Kumkeaw et al., 2013). When is available information about both sensors the covariances of (7) are considered, otherwise the covariances of (8) are considered.

$$
\begin{array}{r}
\mathbf{Q}_{k}=\mathbf{I}(4) \\
\mathbf{R}_{k}=10^{30} \mathbf{I}(3)
\end{array}
$$

In a practical way it means to update the absolute position when both sensors information are available. When the absolute position is not available, it will be considered only the direction indicated by the IMU.

\subsection{Error analysis}

The absolute error of estimation for each time step using (9) will be considered to compare the efficiency of the methods. It consists in the euclidean distance between the estimated position $\left(\hat{x}_{k}, \hat{y}_{k}\right)$ and the reference.

$$
e_{a b s_{k}}=\sqrt{\left(x_{r e f_{k}}-\hat{x}_{k}\right)^{2}+\left(y_{r e f_{k}}-\hat{y}_{k}\right)^{2}}
$$

$x_{r e f_{k}}$ and $y_{r e f_{k}}$ are points of the reference trajectory, here, the trajectory presented in Figure 1. The Root Means Square Error (RMSE) of the absolute error, here called $R M S E_{a b s}$, is computed from absolute error vector $e_{a b s_{k}}$. The sum of the absolute errors here is called accumulated error is $e_{a c}$. The mean of the absolute errors is called average error $e_{\text {avg }}$ and the standard deviation of the absolute errors is $\sigma$.

\section{RESULTS AND DISCUSSIONS}

Next, the comparative results between the two methods are exposed according to the cases indicated on Section 3. The absolute error values for each individual sensor is shown in Figure 2 to observe that, individually, they have large errors. Case 1 deviation characteristics were used.

The estimated trajectory error using only the IMU diverges with time. The integration of the acceleration signal

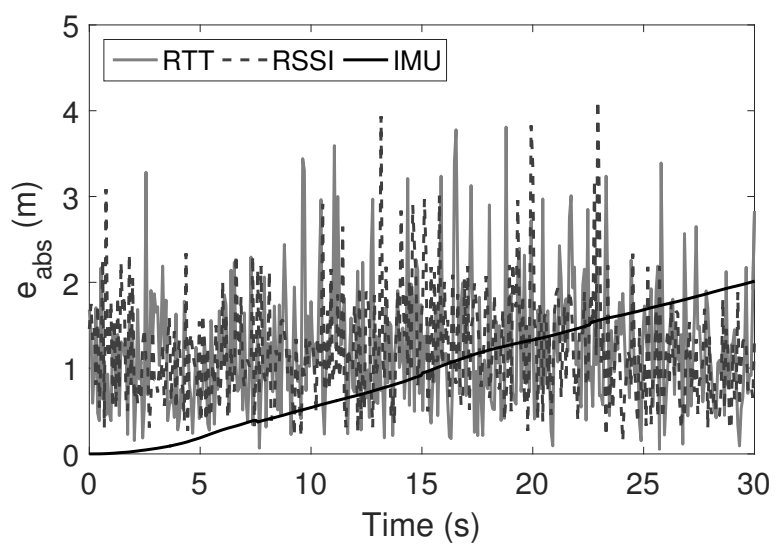

Figure 2. Absolute errors for individual sensors

subjected to a gaussian noise results in a random-walk, whose main characteristic is the growth of variance during time (Spitzer, 2013).

\subsection{Case 1}

Figure 3 shows the absolute error of the estimated trajectory using the EKF fusions IMU+RSSI and IMU+RTT for each time step with $\sigma_{1}$ from Table 1 . It is observed a 2.81 times lower RMSE for the IMU+RTT fusion, as seen in Table 2.

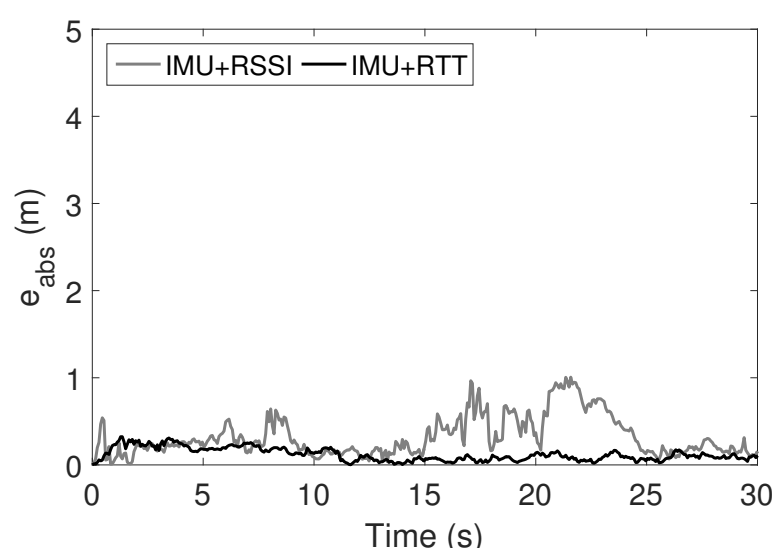

Figure 3. Absolute error for both IMU+RSSI and IMU+RTT fusions for case 1 deviations.

The reference trajectory and the estimations by IMU+RSSI and IMU+RTT fusion are shown in Figure 4. The errors are shown in Table 2.

\begin{tabular}{c|c|c} 
& $I M U+R S S I$ & $I M U+R T T$ \\
\hline$R M S E_{a b s}$ & 0.3938 & 0.1400 \\
\hline$e_{a c}$ & 128.0305 & 47.6404 \\
\hline$e_{a v g}$ & 0.3201 & 0.1191 \\
\hline$\sigma$ & 0.2297 & 0.0736 \\
\hline
\end{tabular}

Table 2. Case 1 fusion errors in meters.

For IMU+RTT fusion the estimated trajectory was more precise, as predicted by the absolute error in Figure 3 . The absolute error of the IMU+RSSI was significantly higher even considering the same standard deviation for both RSSI and RTT measurements. The reason is related with the output functions of both fusion methods. The 


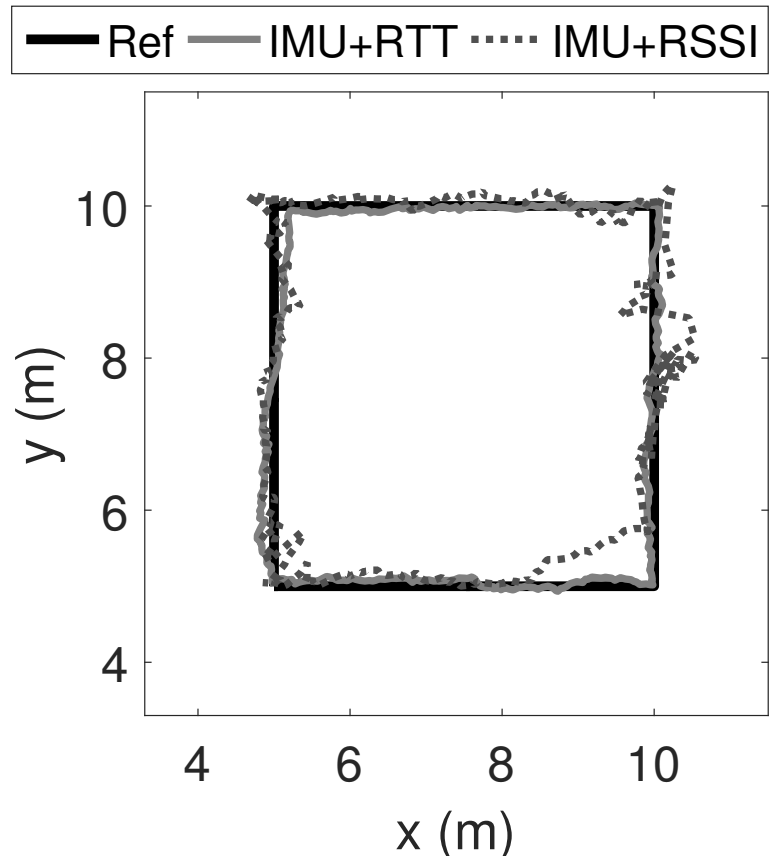

Figure 4. Estimated trajectories for both fusions compared to the reference path considering case 1 deviations.

EKF output of the IMU+RSSI fusion has a log function applied to the euclidean distance measure of both fusions. Besides the distance, $\log$ is another nonlinearity and as a consequence, the linearization of these functions by Jacobian matrices for the EKF leads to better results when using IMU+RTT, even with equal standard deviations.

\subsection{Case 2}

After insert the knowledge about the typical standard deviation for each RSSI and RTT sensors according to Table 1, the simulation was executed again. Figure 5 shows the absolute error during the trajectory for those conditions and Figure 6 shows the trajectory for both fusions and the reference trajectory. Errors are presented in Table 3.

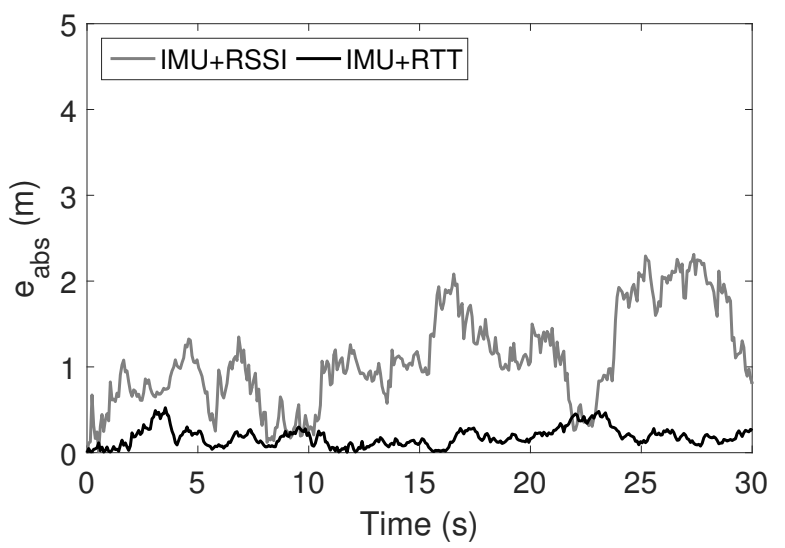

Figure 5. Absolute error for both IMU+RSSI and IMU+RTT fusions for case 2 deviations.

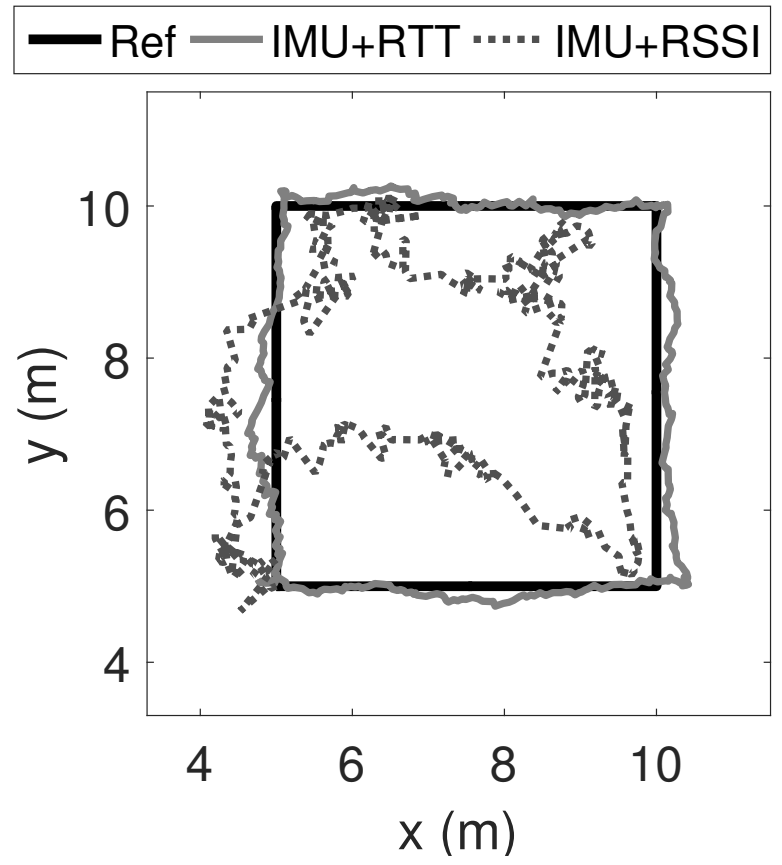

Figure 6. Estimated trajectories for both fusions compared to the reference path considering case 2 deviations.

\begin{tabular}{c|c|c} 
& $I M U+R S S I$ & $I M U+R T T$ \\
\hline$R M S E_{a b s}$ & 1.2279 & 0.2101 \\
\hline$e_{a c}$ & 438.0301 & 72.0681 \\
\hline$e_{a v g}$ & 1.0951 & 0.1802 \\
\hline$\sigma$ & 0.5561 & 0.1081
\end{tabular}

Table 3. Case 2 fusion errors in meters

As exposed in Section 1, the associated errors in the IMU+RTT fusion are intrinsically less expressive than IMU+RSSI errors. This leads to a more accurate trajectory after the EKF fusion.

\subsection{Case 3}

Considering different sample rates for the absolute position sensors and the IMU, it was possible to observe how the EKF fusion would behave in a real experiment. Figure 7 shows the trajectories for both IMU+RTT and IMU+RSSI fusions for multirate case.

The trajectories behaviour translates the chosen methodology used to incorporate the multirate sensors problem in the fusion. It is possible to observe the update of the absolute position in the samples where both informations were available. Between updates the trajectory follows the direction indicated by the accelerometer untill the next absolute position measure. The inefficiency of the IMU+RSSI fusion is more expressive when the multirate case is considered, showing again the practical limitations of this method for small area trajectories. Table 4 exposes these errors. 


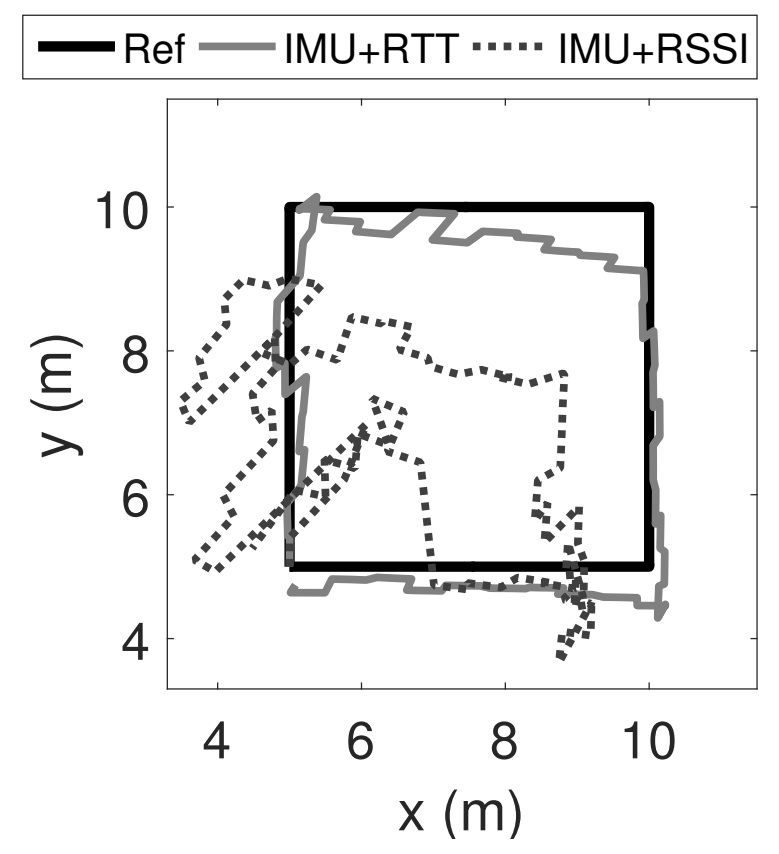

Figure 7. Estimated trajectories compared to the reference path considering multirate sensors $T_{a b s}=10 T_{i m u}$.

\begin{tabular}{c|c|c} 
& $I M U+R S S I$ & $I M U+R T T$ \\
\hline$R M S E_{a b s}$ & 1.9288 & 0.5432 \\
\hline$e_{a c}$ & 692.1232 & 188.7050 \\
\hline$e_{a v g}$ & 1.7303 & 0.4718 \\
\hline$\sigma$ & 0.8532 & 0.2696
\end{tabular}

Table 4 . Case 3 fusion errors in meters.

\section{CONCLUSIONS}

This work presented an approach for EKF based sensor fusion applied to the indoor tracking issue. Considering the characteristics of the new RTT protocol, simulations were made to show the efficiency of this technology in detriment to classical RSSI methods. Results showed that IMU+RTT fusion brings better results than IMU+RSSI, even considering equal standard deviations for both RSSI and RTT measurements. When considered typical standard deviations for each absolute position method, the compared efficiency of IMU+RTT is even better. Also, a multirate case was considered and the IMU+RTT fusion showed acceptable errors. As future work we suggest to implement both fusions in real-time to validate the theory.

\section{ACKNOWLEDGMENTS}

This work was supported by CNPq and FAPESB through project RED0004/2014, Aplicações de Controle em Sistemas Robóticos Não-Holonômicos.

\section{REFERENCES}

Borenstein, J., Everett, H.R., Feng, L., and Wehe, D. (1997). Mobile robot positioning: Sensors and techniques. Journal of robotic systems, 14(4), 231-249.

Brown, R. (2012). Introduction to random signals and applied Kalman filtering : with MATLAB exercises. John Wiley, Hoboken, NJ.

Jin, X.B., Su, T.L., Kong, J.L., Bai, Y.T., Miao, B.B., and Dou, C. (2018). State-of-the-art mobile intelligence: Enabling robots to move like humans by estimating mobility with artificial intelligence. Applied Sciences, 8(3), 379. doi:10.3390/app8030379. URL https://doi. org/10.3390/app8030379.

Julier, S.J. and Uhlmann, J.K. (1997). New extension of the kalman filter to nonlinear systems. In Signal processing, sensor fusion, and target recognition VI, volume 3068, 182-194. International Society for Optics and Photonics.

Kumkeaw, W., Malyavej, V., and Aorpimai, M. (2013). Indoor localization based on multi-rate imu/rssi sensor fusion. ENGINEERING TRANSACTIONS, 16(2), 35.

Mao, G., Anderson, B.D., and Fidan, B. (2007). Path loss exponent estimation for wireless sensor network localization. Computer Networks, 51(10), 2467-2483.

Miranda, J., Abrishambaf, R., Gomes, T., Gonçalves, P., Cabral, J., Tavares, A., and Monteiro, J. (2013). Path loss exponent analysis in wireless sensor networks: Experimental evaluation. In 2013 11th IEEE International Conference on Industrial Informatics (INDIN), 54-58. IEEE.

Nussenzveig, H. (2013). Curso de física básica, 1: mecânica. v. 1. E. Blucher.

Park, S., Ahn, H.S., and Yu, W. (2007). Round-trip time-based wireless positioning without time synchronization. In 2007 International Conference on Control, Automation and Systems. IEEE. doi:10.1109/ iccas.2007.4406716. URL https://doi.org/10.1109/ iccas. 2007.4406716.

Ristic, B., Arulampalam, S., and Gordon, N. (2003). Beyond the Kalman Filter: Particle Filters for Tracking Applications. Artech House. URL https://books. google. com. br/books?id=zABIY--qk2AC.

Ryo Saito, K.I. (2017). Accurate rssi-based indoor localization using time-domain path loss compensation. Eighth International Conference On Indoor Positioning and Indoor Navigation.

Santana, B., Schnitman, L., Ushikoshi, T., and Chagas, T. (2017). Fusão de sensores aplicada à localização de robôs móveis utilizando sistema especialista fuzzy.

Spitzer, F. (2013). Principles of random walk, volume 34. Springer Science \& Business Media.

Want, R., Wang, W., and Chesnutt, S. (2018). Accurate indoor location for the iot. Computer, 51(8), 66-70.

Zhang, D., Xia, F., Yang, Z., Yao, L., and Zhao, W. (2010). Localization technologies for indoor human tracking. In 2010 5th International Conference on Future Information Technology. IEEE. doi:10.1109/ futuretech.2010.5482731. URL https://doi.org/10. 1109/futuretech.2010.5482731. 